\title{
Synthesis of Bicyclic $p$-Diiodobenzenes via Silver-Catalyzed Csp-H Iodination and Ruthenium-Catalyzed Cycloaddition
}

\author{
Yoshihiko Yamamoto, * Kozo Hattori, and Hisao Nishiyama \\ Department of Applied Chemistry, Graduate School of Engineering, Nagoya University, Chikusa,
}

Nagoya 464-8603, JapanExperimental Section

General Considerations. Column chromatography were performed on silica gel (Cica silica gel $60 \mathrm{~N}$ ) with mixed solvents [hexane / ethyl acetate]. ${ }^{1} \mathrm{H}$ and ${ }^{13} \mathrm{C}$ NMR spectra were obtained for samples in $\mathrm{CDCl}_{3}$ solution at $25{ }^{\circ} \mathrm{C}$. ${ }^{1} \mathrm{H} \mathrm{NMR}$ chemical shifts are reported in terms of chemical shift $(\delta, \mathrm{ppm})$ relative to the singlet at $7.26 \mathrm{ppm}$ for chloroform. Splitting patterns are designated as follows: s, singlet; d, doublet; t, triplet; q, quartet; quint, quintet; sext, sextet; sept, septet; m, multiplet. Coupling constants are reported in Hz. ${ }^{13} \mathrm{C}$ NMR spectra were fully decoupled and are reported in terms of chemical shift $(\delta, \mathrm{ppm})$ relative to the triplet at $\delta=77.0 \mathrm{ppm}$ for $\mathrm{CDCl}_{3}$. Elemental analyses were performed by the Instrumental Analysis Facility of Nagoya University. Melting points were obtained in capillary tubes and are uncorrected. 1,2-Dichloroethane and toluene were distilled from $\mathrm{CaH}_{2}$, and degassed before use. Dry DMF was purchased and used directly as received. $\mathrm{Cp} * \mathrm{RuCl}(\mathrm{cod})$ was prepared according to the literature procedures (Oshima, N., Suzuki, H. Moro-oka, Y. Chem. Lett. 1984, 1161-1164.). 
General Procedure for Silver-Catalyzed Iodination (Bromination) of Diynes: Synthesis of diiododiyne 3a-I. A solution of dimethyl dipropargylmalonate (2a) (208.3 mg, $1.0 \mathrm{mmol}$ ), $\mathrm{N}$-iodosuccinimide $(675.2 \mathrm{mg}, 3.0 \mathrm{mmol})$, and $\mathrm{AgNO}_{3}(16.8 \mathrm{mg}, 0.099 \mathrm{mmol})$ in dry $\mathrm{DMF}(6.0 \mathrm{~mL})$ was stirred for $3 \mathrm{~h}$ at room temperature in the dark. The reaction mixture was then quenched with sat. $\mathrm{Na}_{2} \mathrm{~S}_{2} \mathrm{O}_{3}(50 \mathrm{~mL})$, and extracted with AcOEt $(20 \mathrm{~mL} \times 3)$. The combined organic layer was washed with distilled water $(40 \mathrm{~mL} \times 2)$ and brine $(40 \mathrm{~mL} \times 2)$, and dried with $\mathrm{MgSO}_{4}$. The solvent was removed in vacuo and the residue was purified by flash column chromatography on silica gel eluted with hexane/AcOEt (20:1 10:1) to give 3a-I (422.7 mg, 92\%) as colorless solids (mp. 94.9-95. $\left.{ }^{\circ} \mathrm{C}\right): \quad \operatorname{IR}\left(\mathrm{CHCl}_{3}\right) 2253(\mathrm{C} \equiv \mathrm{C}), 1739(\mathrm{C}=\mathrm{O}) \mathrm{cm}^{-1} ; \quad{ }^{1} \mathrm{H}$ NMR $\left(300 \mathrm{MHz}, \mathrm{CDCl}_{3}\right): \delta 3.13$ $(\mathrm{s}, 4 \mathrm{H}), 3.76(\mathrm{~s}, 6 \mathrm{H}) ;{ }^{13} \mathrm{C} \mathrm{NMR}\left(75 \mathrm{MHz}, \mathrm{CDCl}_{3}\right): \delta-2.0,25.2,53.3,56.8,88.2,168.6 ; \quad \mathrm{MS}$ (EI): $m / z(\%): 460(8)\left[\mathrm{M}^{+}\right], 429(20)\left[\mathrm{M}^{+}-\mathrm{OCH}_{3}\right], 401(15)\left[\mathrm{M}^{+}-\mathrm{CO}_{2} \mathrm{Me}\right], 301(100)\left[\mathrm{M}^{+}-\mathrm{HOCH}_{3}\right.$ - I]; EA calcd (\%) for $\mathrm{C}_{11} \mathrm{H}_{10} \mathrm{I}_{2} \mathrm{O}_{4}$ (460.00): C 28.72, H 2.19; found: C 28.89, H 2.22.

3a-Br: oil; $\quad$ IR $\left(\mathrm{CHCl}_{3}\right) 2253(\mathrm{C} \equiv \mathrm{C}), 1736(\mathrm{C}=\mathrm{O}) \mathrm{cm}^{-1} ; \quad{ }^{1} \mathrm{H}$ NMR $\left(300 \mathrm{MHz}, \mathrm{CDCl}_{3}\right)$ : $\delta 2.95(\mathrm{~s}$, $4 \mathrm{H}), 3.73(\mathrm{~s}, 6 \mathrm{H}) ; \quad{ }^{13} \mathrm{C} \mathrm{NMR}\left(75 \mathrm{MHz}, \mathrm{CDCl}_{3}\right): \delta 24.0,42.2,53.2,56.3,74.2,168.5 ; \quad \mathrm{MS}(\mathrm{EI})$ : $m / z(\%): 366$ (4) $\left[\mathrm{M}^{+}\right], 334$ (100) $\left[\mathrm{M}^{+}-\mathrm{HOCH}_{3}\right], 306$ (48) $\left[\mathrm{M}^{+}-\mathrm{H}-\mathrm{CO}_{2} \mathrm{Me}\right], 227$ (86) $\left[\mathrm{M}^{+}-\mathrm{H}\right.$ $-\mathrm{CO}_{2} \mathrm{Me}-\mathrm{Br}$ ]; $\quad$ EA calcd (\%) for $\mathrm{C}_{11} \mathrm{H}_{10} \mathrm{Br}_{2} \mathrm{O}_{4}$ (366.00): C 36.10, H 2.75; found: C 36.02, H 2.83.

3b: mp. $120.0-120.3{ }^{\circ} \mathrm{C} ; \quad \operatorname{IR}\left(\mathrm{CHCl}_{3}\right) 2200(\mathrm{C} \equiv \mathrm{C}) \mathrm{cm}^{-1} ; \quad{ }^{1} \mathrm{H}$ NMR $\left(300 \mathrm{MHz}, \mathrm{CDCl}_{3}\right): \delta 3.20(\mathrm{~s}$, $4 \mathrm{H}) ; \quad{ }^{13} \mathrm{C} \mathrm{NMR}\left(75 \mathrm{MHz}, \mathrm{CDCl}_{3}\right): \delta 5.3,29.4,36.1,83.6,113.1 ; \quad \mathrm{MS}(\mathrm{EI}): m / z(\%): 394(2)\left[\mathrm{M}^{+}\right]$, $267(100)\left[\mathrm{M}^{+}-\mathrm{I}\right], 240(24)\left[\mathrm{M}^{+}-\mathrm{I}-\mathrm{HCN}\right] ; \quad$ EA calcd (\%) for $\mathrm{C}_{9} \mathrm{H}_{4} \mathrm{I}_{2} \mathrm{~N}_{2}$ (393.95): C 27.44, H 1.02, N 7.11; found: C 27.61, H 0.78, N 6.84.

3c: mp. 63.8-64.1 ${ }^{\circ} \mathrm{C} ; \quad$ IR $\left(\mathrm{CHCl}_{3}\right) 2189(\mathrm{C} \equiv \mathrm{C}) \mathrm{cm}^{-1} ; \quad{ }^{1} \mathrm{H}$ NMR $\left(300 \mathrm{MHz}, \mathrm{CDCl}_{3}\right): \delta 4.39(\mathrm{~s}, 4$ $\mathrm{H}) ; \quad{ }^{13} \mathrm{C} \mathrm{NMR}\left(75 \mathrm{MHz}, \mathrm{CDCl}_{3}\right): \delta 4.0,58.2,89.5 ; \quad \mathrm{MS}(\mathrm{EI}): m / z(\%): 345$ (4) $\left[\mathrm{M}^{+}\right], 317(18)\left[\mathrm{M}^{+}\right.$

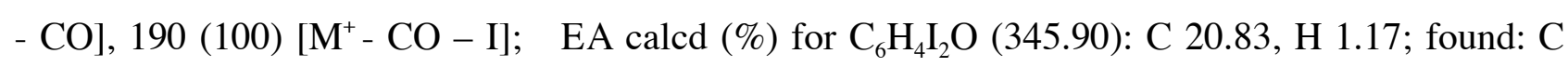
20.99, H 1.22. 
3d: $\quad$ mp. 138.0-138.6 ${ }^{\circ} \mathrm{C} ; \quad \mathrm{IR}\left(\mathrm{CHCl}_{3}\right) 2253(\mathrm{C} \equiv \mathrm{C}), 1354\left(\mathrm{SO}_{2} \mathrm{~N}\right), 1164\left(\mathrm{SO}_{2} \mathrm{~N}\right) \mathrm{cm}^{-1} ; \quad{ }^{1} \mathrm{H}$ NMR $\left(300 \mathrm{MHz}, \mathrm{CDCl}_{3}\right): \delta 2.44(\mathrm{~s}, 3 \mathrm{H}), 4.26(\mathrm{~s}, 4 \mathrm{H}), 7.33$ (d, J = 8.1 Hz, $\left.2 \mathrm{H}\right), 7.69$ (d, J = 8.1 Hz, 2 $\mathrm{H}) ; \quad{ }^{13} \mathrm{C}$ NMR $\left(75 \mathrm{MHz}, \mathrm{CDCl}_{3}\right): \delta 2.7,21.7,38.6,86.3,127.8,129.6,134.6,144.0 ; \quad \mathrm{MS}(\mathrm{EI})$ : $m / z(\%): 499(2)\left[\mathrm{M}^{+}\right], 372(8)\left[\mathrm{M}^{+}-\mathrm{I}\right], 343(100)\left[\mathrm{M}^{+}-\mathrm{H}-\mathrm{SO}_{2} \mathrm{C}_{6} \mathrm{H}_{4} \mathrm{Me}\right] ; \quad$ EA calcd (\%) for $\mathrm{C}_{13} \mathrm{H}_{11} \mathrm{I}_{2} \mathrm{NO}_{2} \mathrm{~S}$ (499.11): C 31.28, H 2.22, N 2.81; found: C 31.33, H 1.96, N 2.52.

3e: $\quad$ mp. $124.1-124.5{ }^{\circ} \mathrm{C} ; \quad \operatorname{IR}\left(\mathrm{CHCl}_{3}\right) 2252(\mathrm{C} \equiv \mathrm{C}) \mathrm{cm}^{-1} ; \quad{ }^{1} \mathrm{H}$ NMR $\left(300 \mathrm{MHz}, \mathrm{CDCl}_{3}\right): \delta 1.40(\mathrm{~s}$, $6 \mathrm{H}), 2.57$ (s, $4 \mathrm{H}), 3.72(\mathrm{~s}, 4 \mathrm{H}) ;{ }^{13} \mathrm{C} \mathrm{NMR}\left(75 \mathrm{MHz}, \mathrm{CDCl}_{3}\right): \delta$-3.3, 23.8, 25.2, 36.1, 65.8, 89.8, 98.2; MS (EI): $m / z(\%): 445(6)\left[\mathrm{M}^{+}\right], 429(100)\left[\mathrm{M}^{+}-\mathrm{CH}_{3}\right], 355(15)\left[\mathrm{M}^{+}-\mathrm{OC}(\mathrm{Me})_{2} \mathrm{O}\right] ; \quad \mathrm{EA}$ calcd (\%) for $\mathrm{C}_{12} \mathrm{H}_{14} \mathrm{I}_{2} \mathrm{O}_{2}$ (444.05): C 32.46, H 3.18; found: C 32.64, H 3.16.

3f: $\quad$ mp. 49.7-49.8 ${ }^{\circ} \mathrm{C} ; \quad \operatorname{IR}\left(\mathrm{CHCl}_{3}\right) 2251(\mathrm{C} \equiv \mathrm{C}) \mathrm{cm}^{-1} ; \quad{ }^{1} \mathrm{H}$ NMR $\left(300 \mathrm{MHz}, \mathrm{CDCl}_{3}\right): \delta 2.58(\mathrm{~s}, 4$ $\mathrm{H}), 3.44(\mathrm{~s}, 4 \mathrm{H}), 4.51(\mathrm{~s}, 4 \mathrm{H}), 7.29-7.37(\mathrm{~m}, 10 \mathrm{H}) ;{ }^{13} \mathrm{C} \mathrm{NMR}\left(75 \mathrm{MHz}, \mathrm{CDCl}_{3}\right): \delta-4.6,24.5$, 42.9, 71.0, 73.6, 90.8, 127.2, 127.3, 128.2, 138.2; MS (EI): $m / z(\%): 584(100)\left[\mathrm{M}^{+}\right], 493(51)\left[\mathrm{M}^{+}\right.$ - $\left.\mathrm{CH}_{2} \mathrm{Ph}\right], 457$ (56) [M+ - I]; EA calcd (\%) for $\mathrm{C}_{23} \mathrm{H}_{22} \mathrm{I}_{2} \mathrm{O}_{2}$ (584.23): C 47.28, H 3.80; found: C 47.31, H 3.77.

3g: $\quad$ mp. 156.5-156.9 ${ }^{\circ} \mathrm{C} ; \quad \operatorname{IR}\left(\mathrm{CHCl}_{3}\right) 2252(\mathrm{C} \equiv \mathrm{C}) \mathrm{cm}^{-1} ; \quad{ }^{1} \mathrm{H}$ NMR $\left(300 \mathrm{MHz}, \mathrm{CDCl}_{3}\right): \delta 2.97(\mathrm{~s}$, $4 \mathrm{H}), 7.34$ (dt, $J=7.5,1.5 \mathrm{~Hz}, 2 \mathrm{H}), 7.41$ (dt, $J=7.5,1.2 \mathrm{~Hz}, 2 \mathrm{H}), 7.66$ (ddd, $J=6.9,1.5,0.6 \mathrm{~Hz}, 2$ $\mathrm{H}), 7.73(\mathrm{ddd}, J=6.9,1.2,0.9 \mathrm{~Hz}, 2 \mathrm{H}) ;{ }^{13} \mathrm{C} \mathrm{NMR}\left(75 \mathrm{MHz}, \mathrm{CDCl}_{3}\right): \delta-3.5,29.7,50.6,91.4$, $119.9,123.8,127.3,128.0,139.6,147.9 ; \quad \mathrm{MS}(\mathrm{EI}): \mathrm{m} / z(\%): 494(19)\left[\mathrm{M}^{+}\right], 329(35)\left[\mathrm{M}^{+}-\right.$ $\left.\mathrm{CH}_{2} \mathrm{C} \equiv \mathrm{CI}\right], 202$ (100) $\left[\mathrm{M}^{+}-\mathrm{CH}_{2} \mathrm{C} \equiv \mathrm{CI}-\mathrm{I}\right] ; \quad$ EA calcd (\%) for $\mathrm{C}_{19} \mathrm{H}_{12} \mathrm{I}_{2}$ (494.11): C 46.18, $\mathrm{H}$ 2.45; found: C 46.26, H 2.56 .

3h: mp. 47.3-47.5 ${ }^{\circ} \mathrm{C} ; \quad$ IR $\left(\mathrm{CHCl}_{3}\right) 2252(\mathrm{C} \equiv \mathrm{C}), 2181(\mathrm{C} \equiv \mathrm{C}) \mathrm{cm}^{-1} ;{ }^{1} \mathrm{H}$ NMR $(300 \mathrm{MHz}$, $\left.\mathrm{CDCl}_{3}\right): \delta 1.83(\mathrm{t}, J=2.4 \mathrm{~Hz}, 3 \mathrm{H}), 4.16(\mathrm{q}, J=2.4 \mathrm{~Hz}, 2 \mathrm{H}), 4.34(\mathrm{~s}, 2 \mathrm{H}) ;{ }^{13} \mathrm{C} \mathrm{NMR}(75 \mathrm{MHz}$, $\left.\mathrm{CDCl}_{3}\right): \delta 3.5,3.7,57.2,57.8,74.1,83.3,89.8 ; \quad \mathrm{MS}(\mathrm{EI}): \mathrm{m} / z(\%): 234(100)\left[\mathrm{M}^{+}\right], 179(74)\left[\mathrm{M}^{+}-\right.$ $2 \mathrm{H}-\mathrm{CH}_{2} \mathrm{C} \equiv \mathrm{CMe}$; $\quad$ EA calcd (\%) for $\mathrm{C}_{7} \mathrm{H}_{7} \mathrm{IO}$ (234.03): C 35.92, H 3.01; found: C 35.98, H 2.94.

3i: oil; $\quad$ IR $\left(\mathrm{CHCl}_{3}\right) 2237(\mathrm{C} \equiv \mathrm{C}), 2184(\mathrm{C} \equiv \mathrm{C}) \mathrm{cm}^{-1} ; \quad{ }^{1} \mathrm{H}$ NMR $\left(300 \mathrm{MHz}, \mathrm{CDCl}_{3}\right): \delta 4.47(\mathrm{~s}, 2$ 
$\mathrm{H}), 4.48(\mathrm{~s}, 2 \mathrm{H}), 7.30-7.35(\mathrm{~m}, 3 \mathrm{H}), 7.45-7.49(\mathrm{~m}, 2 \mathrm{H}) ; \quad{ }^{13} \mathrm{C} \mathrm{NMR}\left(75 \mathrm{MHz}, \mathrm{CDCl}_{3}\right): \delta$ 4.0, 57.4, 58.0, 84.0, 86.8, 89.7, 122.1, 128.1, 128.4, 131.6; MS (EI): $m / z(\%): 296(4)\left[\mathrm{M}^{+}\right], 267(18)\left[\mathrm{M}^{+}-\right.$ $\mathrm{H}-\mathrm{CO}], 139$ (100) $\left[\mathrm{M}^{+}-2 \mathrm{H}-\mathrm{CO}-\mathrm{I}\right] ; \quad$ EA calcd (\%) for $\mathrm{C}_{12} \mathrm{H}_{9} \mathrm{IO}$ (296.10): C 48.67, H 3.06; found: C 48.40, H 2.98 .

3j: $\quad$ mp. 47.3-47.5 ${ }^{\circ} \mathrm{C} ; \quad$ IR $\left(\mathrm{CHCl}_{3}\right) 2253(\mathrm{C} \equiv \mathrm{C}), 2189(\mathrm{C} \equiv \mathrm{C}), 1715\left(\mathrm{CO}_{2} \mathrm{Me}\right) \mathrm{cm}^{-1} ; \quad{ }^{1} \mathrm{H}$ NMR (300 MHz, $\left.\mathrm{CDCl}_{3}\right): \delta 3.78(\mathrm{~s}, 3 \mathrm{H}), 4.37(\mathrm{~s}, 2 \mathrm{H}), 4.40(\mathrm{~s}, 2 \mathrm{H}) ;{ }^{13} \mathrm{C} \mathrm{NMR}\left(75 \mathrm{MHz}, \mathrm{CDCl}_{3}\right): \delta 4.8$, 52.9, 56.2, 58.5, 78.2, 82.5, 88.9, 153.2; $\mathrm{MS}(\mathrm{EI}): \mathrm{m} / z(\%): 278$ (13) $\left[\mathrm{M}^{+}\right], 263$ (28) $\left[\mathrm{M}^{+}-\mathrm{CH}_{3}\right]$, $254(100)\left[\mathrm{M}^{+}-\mathrm{C} \equiv \mathrm{C}\right], 219(10)\left[\mathrm{M}^{+}-\mathrm{CO}_{2} \mathrm{Me}\right] ; \quad$ EA calcd (\%) for $\mathrm{C}_{8} \mathrm{H}_{7} \mathrm{IO}_{3}$ (278.04): C 34.56, $\mathrm{H}$ 2.54; found: C 34.50, H 2.49.

7: $\quad$ mp. 72.5-72.9 ${ }^{\circ} \mathrm{C} ; \quad \operatorname{IR}\left(\mathrm{CHCl}_{3}\right) 2252(\mathrm{C} \equiv \mathrm{C}), 2187(\mathrm{C} \equiv \mathrm{C}) \mathrm{cm}^{-1} ; \quad{ }^{1} \mathrm{H}$ NMR $\left(300 \mathrm{MHz}, \mathrm{CDCl}_{3}\right)$ : $\delta 4.26(\mathrm{~s}, 4 \mathrm{H}), 4.36(\mathrm{~s}, 4 \mathrm{H}) ; \quad{ }^{13} \mathrm{C} \mathrm{NMR}\left(75 \mathrm{MHz} \mathrm{CDCl}_{3}\right): \delta 4.2,56.8,58.0,82.0,89.4 ; \quad \mathrm{MS}(\mathrm{EI})$ : $m / z(\%): 414(13)\left[\mathrm{M}^{+}\right] ; \quad$ EA calcd (\%) for $\mathrm{C}_{10} \mathrm{H}_{8} \mathrm{I}_{2} \mathrm{O}_{2}$ (413.98): C 29.01, $\mathrm{H} \mathrm{1.95}$; found: C 29.00, $\mathrm{H}$ 1.96.

15: mp. 109.0-109.5 ${ }^{\circ} \mathrm{C} ; \quad \operatorname{IR}\left(\mathrm{CHCl}_{3}\right) 1740\left(\mathrm{CO}_{2} \mathrm{Me}\right) \mathrm{cm}^{-1} ; \quad{ }^{1} \mathrm{H}$ NMR $\left(300 \mathrm{MHz}, \mathrm{CDCl}_{3}\right): \delta 3.01$ (s, $4 \mathrm{H}), 3.09$ (s, $4 \mathrm{H}), 3.74(\mathrm{~s}, 6 \mathrm{H}) ;{ }^{13} \mathrm{C} \mathrm{NMR}\left(75 \mathrm{MHz}, \mathrm{CDCl}_{3}\right): \delta$-1.6, 23.7, 25.2, 53.3, 56.5,

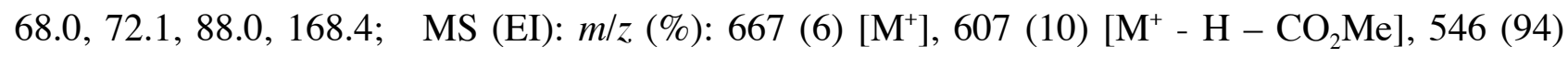
$\left[\mathrm{M}^{+}-3 \mathrm{H}-2 \mathrm{CO}_{2} \mathrm{Me}\right], 487(78)\left[\mathrm{M}^{+}-3 \mathrm{H}-3 \mathrm{CO}_{2} \mathrm{Me}\right], 419(100)\left[\mathrm{M}^{+}-3 \mathrm{H}-2 \mathrm{CO}_{2} \mathrm{Me}-\mathrm{I}\right] ; \quad \mathrm{EA}$ calcd (\%) for $\mathrm{C}_{22} \mathrm{H}_{20} \mathrm{I}_{2} \mathrm{O}_{8}$ (666.20): C 39.66, H 3.03; found: C 39.78, H 3.10.

General Procedure for Ruthenium-Catalyzed Cycloaddition with Acetylene: Synthesis of

Diiodobenzene 4a-I. To a solution of $\mathrm{Cp} * \mathrm{RuCl}(\operatorname{cod})(5.7 \mathrm{mg}, 0.015 \mathrm{mmol})$ in dry degassed 1,2-dichloroethane $(1.5 \mathrm{~mL})$ was added a solution of diiododiyne 3a-I (138.2 $\mathrm{mg}, 0.30 \mathrm{mmol})$ in dry degassed 1,2-dichloroethane $(2.0 \mathrm{~mL})$ over $15 \mathrm{~min}$ at room temperature under acetylene atmosphere and the solution was stirred for $30 \mathrm{~min}$. The solvent was removed in vacuo, and the residue was purified by flash column chromatography on silica gel eluted with hexane/AcOEt 20:1) to give 4a-I (120.0 mg, 83\%) as colorless solids (mp. 131.9-132.2 $\left.{ }^{\circ} \mathrm{C}\right): \quad$ IR $\left(\mathrm{CHCl}_{3}\right) 1735\left(\mathrm{CO}_{2} \mathrm{Me}\right) \mathrm{cm}^{-1} ; \quad{ }^{1} \mathrm{H}$ 
NMR (300 MHz, $\left.\mathrm{CDCl}_{3}\right): \delta 3.70(\mathrm{~s}, 4 \mathrm{H}), 3.79(\mathrm{~s}, 6 \mathrm{H}), 7.25(\mathrm{~s}, 2 \mathrm{H}) ;{ }^{13} \mathrm{C} \mathrm{NMR}(75 \mathrm{MHz}$, $\left.\mathrm{CDCl}_{3}\right): \delta 47.03,53.29,56.57,92.44,137.71,144.63,171.13 ; \quad \mathrm{MS}(\mathrm{EI}): \mathrm{m} / z(\%): 486(72)\left[\mathrm{M}^{+}\right]$, 426 (100) $\left[\mathrm{M}^{+}-\mathrm{H}-\mathrm{CO}_{2} \mathrm{Me}\right], 395$ (20) $\left[\mathrm{M}^{+}-\mathrm{H}-\mathrm{CO}_{2} \mathrm{Me}-\mathrm{OMe}\right.$, 367 (30) $\left[\mathrm{M}^{+}-\mathrm{H}-2 \mathrm{CO}_{2} \mathrm{Me}\right]$; EA calcd (\%) for $\mathrm{C}_{13} \mathrm{H}_{12} \mathrm{I}_{2} \mathrm{O}_{4}$ (486.04): C 32.12, H 2.49; found: C 32.08, H 2.53.

4a-Br: mp. $133.4-133.6{ }^{\circ} \mathrm{C}$ : $\quad \operatorname{IR}\left(\mathrm{CHCl}_{3}\right) 1736\left(\mathrm{CO}_{2} \mathrm{Me}\right) \mathrm{cm}^{-1} ;{ }^{1} \mathrm{H}$ NMR $\left(300 \mathrm{MHz}, \mathrm{CDCl}_{3}\right): \delta$ 3.69 (s, $2 \mathrm{H}), 3.78(\mathrm{~s}, 6 \mathrm{H}), 7.19(\mathrm{~s}, 2 \mathrm{H}) ;{ }^{13} \mathrm{C} \mathrm{NMR}\left(75 \mathrm{MHz}, \mathrm{CDCl}_{3}\right): \delta$ 42.96, 53.29, 57.72, 117.96, 131.62, 141.64, 171.15; $\mathrm{MS}(\mathrm{EI}): \mathrm{m} / z(\%): 392$ (32) $\left[\mathrm{M}^{+}\right], 332$ (100) $\left[\mathrm{M}^{+}-\mathrm{H}-\mathrm{CO}_{2} \mathrm{Me}\right]$, 301 (12) $\left[\mathrm{M}^{+}-\mathrm{H}-\mathrm{CO}_{2} \mathrm{Me}-\mathrm{OMe}\right], 273$ (36) $\left[\mathrm{M}^{+}-\mathrm{H}-2 \mathrm{CO}_{2} \mathrm{Me}\right] ; \quad$ EA calcd (\%) for $\mathrm{C}_{13} \mathrm{H}_{12} \mathrm{Br}_{2} \mathrm{O}_{4}$ (392.04): C 39.83, H 3.09; found: C 39.86, H 3.16.

4b: $\quad$ mp. 176.6-177.0 ${ }^{\circ} \mathrm{C}: \quad{ }^{1} \mathrm{H}$ NMR $\left(300 \mathrm{MHz}, \mathrm{CDCl}_{3}\right): \delta 3.86(\mathrm{~s}, 4 \mathrm{H}), 7.26(\mathrm{~s}, 2 \mathrm{H}) ; \quad{ }^{13} \mathrm{C} \mathrm{NMR}$ $\left(75 \mathrm{MHz}, \mathrm{CDCl}_{3}\right): \delta 30.4,50.6,92.1,115.5,139.3,141.3 ; \quad \mathrm{MS}(\mathrm{EI}): \mathrm{m} / z(\%): 420(100)\left[\mathrm{M}^{+}\right], 293$ (4) $\left[\mathrm{M}^{+}-\mathrm{I}\right], 266$ (4) $\left[\mathrm{M}^{+}-\mathrm{I}-\mathrm{HCN}\right]$; EA calcd (\%) for $\mathrm{C}_{11} \mathrm{H}_{6} \mathrm{I}_{2} \mathrm{~N}_{2}$ (419.99): C 31.46, H 1.44, N 6.67; found: C 31.48, H 1.21, N 6.50.

4c: $\quad$ mp. 117.2-117.5 ${ }^{\circ} \mathrm{C}: \quad{ }^{1} \mathrm{H}$ NMR $\left(300 \mathrm{MHz} \mathrm{CDCl}_{3}\right): \delta 5.12(\mathrm{~s}, 4 \mathrm{H}), 7.29(\mathrm{~s}, 2 \mathrm{H}) ; \quad{ }^{13} \mathrm{C} \mathrm{NMR}$ $\left(75 \mathrm{MHz}, \mathrm{CDCl}_{3}\right): \delta 79.2,86.9,137.7,144.4 ; \quad \mathrm{MS}(\mathrm{EI}): \mathrm{m} / z(\%): 372(78)\left[\mathrm{M}^{+}\right], 344(24)\left[\mathrm{M}^{+}-\right.$ CO], 217 (100) [ $\mathrm{M}^{+}-\mathrm{CO}$ - I]; EA calcd (\%) for $\mathrm{C}_{8} \mathrm{H}_{6} \mathrm{I}_{2} \mathrm{O}$ (371.94): C 25.83, H 1.63; found: C 25.77, H 1.71 .

4d: $\quad$ mp. $217.5-218.0{ }^{\circ} \mathrm{C}: \quad \mathrm{IR}\left(\mathrm{CHCl}_{3}\right) 1350\left(\mathrm{SO}_{2} \mathrm{~N}\right), 1166\left(\mathrm{SO}_{2} \mathrm{~N}\right) \mathrm{cm}^{-1} ;{ }^{1} \mathrm{H}$ NMR $(300 \mathrm{MHz}$, $\left.\mathrm{CDCl}_{3}\right): \delta 2.43(\mathrm{~s}, 3 \mathrm{H}), 4.63(\mathrm{~s}, 4 \mathrm{H}), 7.28(\mathrm{~s}, 2 \mathrm{H}), 7.36(\mathrm{~d}, J=8.5 \mathrm{~Hz}, 2 \mathrm{H}), 7.80(\mathrm{~d}, J=8.5 \mathrm{~Hz}, 2$ $\mathrm{H}) ; \quad{ }^{13} \mathrm{C} \mathrm{NMR}\left(75 \mathrm{MHz}, \mathrm{CDCl}_{3}\right): \delta 21.7,59.6,89.2,127.4,129.9,133.4,138.2,141.5,143.9$;

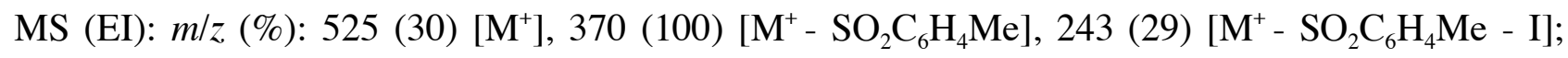
EA calcd (\%) for $\mathrm{C}_{15} \mathrm{H}_{13} \mathrm{I}_{2} \mathrm{NO}_{2} \mathrm{~S}$ (525.14): C 34.31, H 2.50, N 2.67; found: C 34.42, H 2.20 N 2.42.

4e: $\quad$ mp. 149.9-150.2 ${ }^{\circ} \mathrm{C}: \quad{ }^{1} \mathrm{H} \mathrm{NMR}\left(300 \mathrm{MHz}, \mathrm{CDCl}_{3}\right): \delta 1.48(\mathrm{~s}, 6 \mathrm{H}), 2.99(\mathrm{~s}, 4 \mathrm{H}), 3.75(\mathrm{~s}, 4 \mathrm{H})$, $7.24(\mathrm{~s}, 2 \mathrm{H}) ; \quad{ }^{13} \mathrm{C} \mathrm{NMR}\left(75 \mathrm{MHz}, \mathrm{CDCl}_{3}\right): \delta 23.9,38.9,46.9,68.7,93.9,98.0,137.4,146.3 ; \quad \mathrm{MS}$ (EI): $m / z(\%): 470(25)\left[\mathrm{M}^{+}\right], 455(100)\left[\mathrm{M}^{+}-\mathrm{CH}_{3}\right], 395(39)\left[\mathrm{M}^{+}-\mathrm{OC}(\mathrm{Me})_{2} \mathrm{O}\right] ; \quad \mathrm{EA}$ calcd (\%) for 
$\mathrm{C}_{14} \mathrm{H}_{16} \mathrm{I}_{2} \mathrm{O}_{2}$ (470.08): C 35.77, H 3.43; found: C 35.95, H 3.44.

4f: $\quad$ mp. 91.2-91.7 ${ }^{\circ} \mathrm{C}: \quad{ }^{1} \mathrm{H}$ NMR $\left(300 \mathrm{MHz}, \mathrm{CDCl}_{3}\right): \delta 2.99(\mathrm{~s}, 4 \mathrm{H}), 3.48(\mathrm{~s}, 4 \mathrm{H}), 4.53(\mathrm{~s}, 4 \mathrm{H})$, $7.20(\mathrm{~s}, 2 \mathrm{H}), 7.27-7.36(\mathrm{~m}, 10 \mathrm{H}) ;{ }^{13} \mathrm{C} \mathrm{NMR}\left(75 \mathrm{MHz} \mathrm{CDCl}_{3}\right): \delta$ 45.2, 46.0, 73.1, 73.2, 93.8, 127.3, 127.4, 128.2, 137.0, 138.3, 147.1; $\mathrm{MS}(\mathrm{EI}): \mathrm{m} / z(\%): 610$ (11) $\left[\mathrm{M}^{+}\right], 519$ (67) $\left[\mathrm{M}^{+}-\mathrm{CH}_{2} \mathrm{Ph}\right]$, 502 (39) $\left[\mathrm{MH}^{+}-\mathrm{OCH}_{2} \mathrm{Ph}\right], 381$ (100) $\left[\mathrm{MH}^{+}-\mathrm{OCH}_{2} \mathrm{Ph}-\mathrm{CH}_{2} \mathrm{OCH}_{2} \mathrm{Ph}\right] ; \mathrm{EA}$ calcd (\%) for $\mathrm{C}_{25} \mathrm{H}_{24} \mathrm{I}_{2} \mathrm{O}_{2}$ (610.27): C 49.20, H 3.96; found: C 49.23, H 3.90.

4g: $\quad$ mp. 273.2-273.6 ${ }^{\circ} \mathrm{C}: \quad{ }^{1} \mathrm{H}$ NMR (300 MHz, $\left.\mathrm{CDCl}_{3}\right): \delta 3.56(\mathrm{~s}, 4 \mathrm{H})$, 7.25-7.27 (m, $\left.4 \mathrm{H}\right)$, 7.33-7.41 (m, $4 \mathrm{H}), 7.72-7.75(\mathrm{~m}, 2 \mathrm{H}) ;{ }^{13} \mathrm{C} \mathrm{NMR}\left(75 \mathrm{MHz}, \mathrm{CDCl}_{3}\right): \delta 52.1,53.9,93.2,119.9$, 122.2, 127.6, 127.7, 137.6, 139.4, 147.7, 151.5; MS (EI): $m / z(\%): 520$ (100) $\left[\mathrm{M}^{+}\right], 392(4)\left[\mathrm{MH}^{+}-\right.$ I], 265 (56) $\left[\mathrm{MH}^{+}-2 \mathrm{I}\right] ; \quad$ EA calcd (\%) for $\mathrm{C}_{21} \mathrm{H}_{14} \mathrm{I}_{2}$ (520.14): C 48.49, H 2.71; found: C 48.56, $\mathrm{H}$ 2.63 .

4h: $\quad$ mp. $31.3-31.8{ }^{\circ} \mathrm{C}: \quad{ }^{1} \mathrm{H}$ NMR $\left(300 \mathrm{MHz} \mathrm{CDCl}_{3}\right): \delta 2.18(\mathrm{~s}, 3 \mathrm{H}), 5.01(\mathrm{~s}, 2 \mathrm{H}), 5.20(\mathrm{~s}, 2 \mathrm{H})$, $6.79(\mathrm{~d}, J=7.5 \mathrm{~Hz}, 1 \mathrm{H}), 7.49(\mathrm{~d}, J=7.5 \mathrm{~Hz}, 1 \mathrm{H}) ;{ }^{13} \mathrm{C} \mathrm{NMR}\left(75 \mathrm{MHz}, \mathrm{CDCl}_{3}\right): \delta$ 18.4, 74.6, 78.1, 83.4, 130.1, 131.1, 136.2, 139.0, 142.9; $\mathrm{MS}(\mathrm{EI}): \mathrm{m} / \mathrm{z}(\%): 260$ (74) $\left[\mathrm{M}^{+}\right], 245$ (26) $\left[\mathrm{M}^{+}-\mathrm{CH}_{3}\right]$, 231 (34) $\left[\mathrm{MH}^{+}-\mathrm{CO}\right], 217(65)\left[\mathrm{M}^{+}-\mathrm{CH}_{3}-\mathrm{CO}\right], 105(100)\left[\mathrm{M}^{+}-\mathrm{CO}-\mathrm{I}\right]$; EA calcd (\%) for $\mathrm{C}_{9} \mathrm{H}_{9} \mathrm{IO}$ (260.07): C 41.56, H 3.49; found: C 41.67, H 3.38.

4i: $\quad$ mp. 81.2-81.6 ${ }^{\circ} \mathrm{C}: \quad{ }^{1} \mathrm{H}$ NMR $\left(300 \mathrm{MHz}, \mathrm{CDCl}_{3}\right): \delta 5.08$ (br s, $\left.2 \mathrm{H}\right), 5.34$ (br s, $\left.2 \mathrm{H}\right), 7.06(\mathrm{~d}, J$ $=7.8 \mathrm{~Hz}, 1 \mathrm{H}), 7.35-7.47(\mathrm{~m}, 5 \mathrm{H}), 7.70(\mathrm{~d}, J=7.8 \mathrm{~Hz}, 1 \mathrm{H}) ;{ }^{13} \mathrm{C} \mathrm{NMR}\left(75 \mathrm{MHz}, \mathrm{CDCl}_{3}\right): \delta 75.0$, 77.9, 86.1, 127.5, 127.7, 128.7, 129.2, 135.8, 136.8, 137.8, 138.9, 144.1; $\quad \mathrm{MS}(\mathrm{EI}): \mathrm{m} / z(\%): 322$ (100) $\left[\mathrm{M}^{+}\right], 293$ (9) $\left[\mathrm{M}^{+}-\mathrm{H}-\mathrm{CO}\right], 245$ (2) $\left[\mathrm{M}^{+}-\mathrm{C}_{6} \mathrm{H}_{5}\right], 195$ (9) $\left[\mathrm{M}^{+}-\mathrm{I}\right], 165$ (94) $\left[\mathrm{M}^{+}-2 \mathrm{H}-\mathrm{CO}-\right.$ I]; $\quad$ EA calcd (\%) for $\mathrm{C}_{14} \mathrm{H}_{11} \mathrm{IO}$ (322.14): C 52.20, H 3.44; found: C 52.14, H 3.45.

4j: $\quad$ mp. 94.8-95.1 ${ }^{\circ} \mathrm{C}: \quad \operatorname{IR}\left(\mathrm{CHCl}_{3}\right) 1718\left(\mathrm{CO}_{2} \mathrm{Me}\right) \mathrm{cm}^{-1} ; \quad{ }^{1} \mathrm{H}$ NMR $\left(300 \mathrm{MHz}, \mathrm{CDCl}_{3}\right): \delta 3.90(\mathrm{~s}$, $3 \mathrm{H}), 5.01-5.02(\mathrm{~m}, 2 \mathrm{H}), 5.50-5.52(\mathrm{~m}, 2 \mathrm{H}), 7.61$ (d, J = 8.4 Hz, $1 \mathrm{H}), 7.71(\mathrm{~d}, J=8.4 \mathrm{~Hz}, 1 \mathrm{H})$; ${ }^{13} \mathrm{C} \mathrm{NMR}\left(75 \mathrm{MHz}, \mathrm{CDCl}_{3}\right): \delta 52.3,76.5,93.7,124.1,130.1,136.7,142.0,145.1,165.9 ; \quad \mathrm{MS}(\mathrm{EI})$ : $m / z(\%): 304(58)\left[\mathrm{M}^{+}\right], 289(100)\left[\mathrm{M}^{+}-\mathrm{CH}_{3}\right], 244(17)\left[\mathrm{M}^{+}-\mathrm{H}-\mathrm{CO}_{2} \mathrm{Me}\right] ; \quad$ EA calcd (\%) for 
$\mathrm{C}_{10} \mathrm{H}_{9} \mathrm{IO}_{3}$ (304.08): C 39.50, H 2.98; found: C 39.50, H 2.97.

16: mp. 139.4-139.9 ${ }^{\circ} \mathrm{C}$ : $\quad$ IR $\left(\mathrm{CHCl}_{3}\right) 1734\left(\mathrm{CO}_{2} \mathrm{Me}\right) \mathrm{cm}^{-1} ; \quad{ }^{1} \mathrm{H}$ NMR $\left(300 \mathrm{MHz}, \mathrm{CDCl}_{3}\right): \delta 3.49$ (s, $4 \mathrm{H}), 3.62(\mathrm{~s}, 4 \mathrm{H}), 3.73(\mathrm{~s}, 12 \mathrm{H}), 6.76(\mathrm{~d}, J=8.1 \mathrm{~Hz}, 2 \mathrm{H}), 7.61(\mathrm{~d}, J=8.1 \mathrm{~Hz}, 2 \mathrm{H}) ;{ }^{13} \mathrm{C}$ NMR (75 MHz, $\left.\mathrm{CDCl}_{3}\right): \delta 41.0,45.9,53.1,58.12,91.9,128.8,135.8,136.5,138.5,144.6,171.2$; MS (EI): $m / z(\%): 720(100)\left[\mathrm{MH}_{2}^{+}\right], 658(10)\left[\mathrm{M}^{+}-\mathrm{H}-\mathrm{CO}_{2} \mathrm{Me}\right], 626(40)\left[\mathrm{M}^{+}-2 \mathrm{H}-\mathrm{CO}_{2} \mathrm{Me}-\right.$ OMe]; EA calcd (\%) for $\mathrm{C}_{26} \mathrm{H}_{24} \mathrm{I}_{2} \mathrm{O}_{8}$ (718.27): C 43.48, H 3.37; found: $\mathrm{C} 43.34, \mathrm{H} 3.51$.

\section{General Procedure for Ruthenium-Catalyzed Cycloaddition with Monoalkynes: Synthesis of}

Diiodobenzene 5a. To a solution of $\mathrm{Cp} * \mathrm{RuCl}(\mathrm{cod})(5.8 \mathrm{mg}, 0.015 \mathrm{mmol})$ and 1-hexyne (37.0 mg, $0.45 \mathrm{mmol})$ in dry degassed 1,2-dichloroethane $(1.5 \mathrm{~mL})$ was added a solution of diiododiyne $\mathbf{3 c}$ $(103.7 \mathrm{mg}, 0.30 \mathrm{mmol})$ in dry degassed 1,2-dichloroethane $(2.0 \mathrm{~mL})$ over $15 \mathrm{~min}$ at room temperature under argon atmosphere and the solution was stirred for $4 \mathrm{~h}$. The solvent was removed in vacuo, and the residue was purified by flash column chromatography on silica gel eluted with hexane/AcOEt 50:1 20:1) to give 5a $(106.7 \mathrm{mg}, 83 \%)$ as colorless solids (mp. 60.5-60.7 $\left.{ }^{\circ} \mathrm{C}\right): \quad{ }^{~} \mathrm{H}$ NMR (300 MHz, $\left.\mathrm{CDCl}_{3}\right): \delta 0.96(\mathrm{t}, J=7.2 \mathrm{~Hz}, 3 \mathrm{H}), 1.34-1.59(\mathrm{~m}, 4 \mathrm{H}), 2.65(\mathrm{t}, J=7.5 \mathrm{~Hz}, 2 \mathrm{H})$, 5.12 (s, $4 \mathrm{H}), 7.40(\mathrm{~s}, 1 \mathrm{H}) ; \quad{ }^{13} \mathrm{C} \mathrm{NMR}\left(75 \mathrm{MHz}, \mathrm{CDCl}_{3}\right): \delta$ 14.0, 22.5, 32.5, 39.1, 79.2, 80.3, 87.0, 93.2, 136.5, 141.4, 145.2, 146.3; MS (EI): $m / z(\%): 428$ (100) $\left[\mathrm{M}^{+}\right], 399(25)\left[\mathrm{M}^{+}-\mathrm{C}_{2} \mathrm{H}_{5}\right], 385$ (21) $\left[\mathrm{M}^{+}-\mathrm{C}_{3} \mathrm{H}_{7}\right], 357(41)\left[\mathrm{M}^{+}-\mathrm{C}_{3} \mathrm{H}_{7}-\mathrm{CO}\right], 301(25)\left[\mathrm{M}^{+}-\mathrm{I}\right], 273(82)\left[\mathrm{M}^{+}-\mathrm{CO}-\mathrm{I}\right] ; \quad$ EA calcd (\%) for $\mathrm{C}_{12} \mathrm{H}_{14} \mathrm{I}_{2} \mathrm{O}$ (428.05): C 33.67, H 3.30; found: C 33.58, H 3.39.

5b: mp. 135.2-135.4 ${ }^{\circ} \mathrm{C} ; \quad{ }^{1} \mathrm{H}$ NMR $\left(300 \mathrm{MHz}, \mathrm{CDCl}_{3}\right): \delta 5.21(\mathrm{~s}, 4 \mathrm{H}), 7.29-7.32(\mathrm{~m}, 2 \mathrm{H})$, 7.39-7.44 (m, $3 \mathrm{H}), 7.55(\mathrm{~s}, 1 \mathrm{H}) ;{ }^{13} \mathrm{C} \mathrm{NMR}\left(75 \mathrm{MHz}, \mathrm{CDCl}_{3}\right)$ : $\delta$ 79.3, 80.5, 86.9, 91.6, 127.9, 128.0, 129.1, 137.3, 141.7, 142.7, 145.3, 147.2; MS (EI): $\mathrm{m} / \mathrm{z}(\%): 448(100)\left[\mathrm{M}^{+}\right], 419(15)\left[\mathrm{M}^{+}-\right.$ $\mathrm{H}-\mathrm{CO}], 293(36)\left[\mathrm{M}^{+}-\mathrm{CO}-\mathrm{I}\right], 165(72)\left[\mathrm{M}^{+}-\mathrm{H}-\mathrm{CO}-2 \mathrm{I}\right] ; \quad \mathrm{EA}$ calcd $(\%)$ for $\mathrm{C}_{14} \mathrm{H}_{10} \mathrm{I}_{2} \mathrm{O}$ (448.04): C 37.53, H 2.25; found: C 37.70, H 2.25.

5c: $\quad$ mp. 195.8-196.2 ${ }^{\circ} \mathrm{C} ; \quad{ }^{1} \mathrm{H}$ NMR $\left(300 \mathrm{MHz}, \mathrm{CDCl}_{3}\right): \delta 5.29(\mathrm{~s}, 4 \mathrm{H}), 6.93-6.96(\mathrm{~m}, 4 \mathrm{H})$, 7.13-7.21 (m, $6 \mathrm{H}) ;{ }^{13} \mathrm{C} \mathrm{NMR}\left(75 \mathrm{MHz}, \mathrm{CDCl}_{3}\right): \delta 80.8,94.0,127.1,127.4,129.7,143.1,143.7$, 


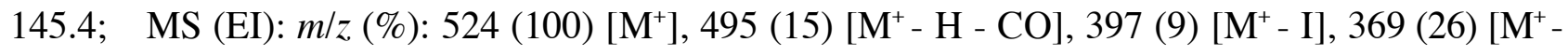
$\mathrm{CO}-\mathrm{I}], 269(5)\left[\mathrm{M}^{+}-\mathrm{H}-2 \mathrm{I}\right], 239(84)\left[\mathrm{M}^{+}-\mathrm{H}-\mathrm{CO}-2 \mathrm{HI}\right] ; \quad$ EA calcd (\%) for $\mathrm{C}_{20} \mathrm{H}_{14} \mathrm{I}_{2} \mathrm{O}(524.13)$ : C 45.83, H 2.69; found: C 45.88, H 2.64.

5d/5d': oil (6:4 mixture); ${ }^{1} \mathrm{H}$ NMR $\left(300 \mathrm{MHz}, \mathrm{CDCl}_{3}\right): \delta 0.95$ and $0.97(\mathrm{t}, J=7.2 \mathrm{~Hz}, 3 \mathrm{H})$, 1.33-1.61 (m, $4 \mathrm{H}), 2.10$ and $2.17(\mathrm{~s}, 3 \mathrm{H}), 2.55$ and $2.68(\mathrm{t}, J=7.8 \mathrm{~Hz}, 2 \mathrm{H}), 4.99-5.02(\mathrm{~m}, 2 \mathrm{H})$, 5.20-5.21 (m, $2 \mathrm{H}), 6.89$ and $7.40(\mathrm{~s}, 1 \mathrm{H}) ;{ }^{13} \mathrm{C} \mathrm{NMR}\left(75 \mathrm{MHz}, \mathrm{CDCl}_{3}\right): \delta 14.1$ and 15.3, 18.2, 22.6 and 22.7, 32.2 and 32.8, 32.7 and 39.3, 74.8 and 75.0, 78.1 and 79.2, 83.5 and 89.4, 129.0 and 129.2, 130.0 and 142.6, 136.1 and 136.6, 139.5 and 140.4, 143.9 and 144.2; MS (EI): $m / z(\%)$ : $316(100)\left[\mathrm{M}^{+}\right], 287(20)\left[\mathrm{M}^{+}-\mathrm{CH}_{2} \mathrm{CH}_{3}\right], 189(12)\left[\mathrm{M}^{+}-\mathrm{I}\right], 161(57)\left[\mathrm{M}^{+}-\mathrm{CH}_{2}=\mathrm{CH}_{2}-\mathrm{I}\right] ; \quad$ EA calcd (\%) for $\mathrm{C}_{13} \mathrm{H}_{17} \mathrm{IO}$ (316.18): C 49.38, H 5.42; found: C 49.14, H 5.27.

Procedure for Ruthenium-Catalyzed Cyclization of Diiotriyne 7. To a solution of $\mathrm{Cp} * \mathrm{RuCl}(\mathrm{cod})(17.1 \mathrm{mg}, 0.045 \mathrm{mmol})$ in dry degassed 1,2-dichloroethane $(1.5 \mathrm{~mL})$ was added a solution of diiodotriyne $7(124.1 \mathrm{mg}, 0.30 \mathrm{mmol})$ in dry degassed 1,2-dichloroethane $(2.0 \mathrm{~mL})$ over $15 \mathrm{~min}$ at room temperature under argon atmosphere and the solution was stirred for $24 \mathrm{~h}$. The solvent was removed in vacuo, and the residue was purified by flash column chromatography on silica gel eluted with hexane/AcOEt 20:1 5:1) to give 8 (72.5 mg, 60\%) as brown solids (mp. 219.8-220.4 $\left.{ }^{\circ} \mathrm{C}\right): \quad{ }^{1} \mathrm{H}$ NMR $\left(300 \mathrm{MHz}, \mathrm{CDCl}_{3}\right): \delta 5.00(\mathrm{~s}, 4 \mathrm{H}), 5.14(\mathrm{~s}, 4 \mathrm{H}) ; \quad{ }^{13} \mathrm{C} \mathrm{NMR}(75 \mathrm{MHz}$, $\left.\mathrm{CDCl}_{3}\right): \delta 73.6,79.9,80.3,99.4,132.4,145.8 ; \quad \mathrm{MS}(\mathrm{EI}): \mathrm{m} / z(\%): 414(100)\left[\mathrm{M}^{+}\right], 287(12)\left[\mathrm{M}^{+}-\right.$ I]; EA calcd (\%) for $\mathrm{C}_{10} \mathrm{H}_{8} \mathrm{I}_{2} \mathrm{O}_{2}$ (413.98): C 29.01, H 1.95; found: C 29.14, H 1.92.

Procedure of Mizoroki-Heck Reaction of 4e. A solution of $\mathrm{Pd}_{2}(\mathrm{dba})_{3} \bullet \mathrm{CHCl}_{3}(7.8 \mathrm{mg}, 0.0075$ mmol), SPhos (13.6 mg, $0.033 \mathrm{mmol}), 4 \mathrm{e}(141.0 \mathrm{mg}, 0.30 \mathrm{mmol})$, and styrene (125.0 $\mathrm{mg}, 1.20$ mmol) in degassed mixed solvent (DMF $5 \mathrm{~mL} / \mathrm{Et}_{3} \mathrm{~N} 1 \mathrm{~mL}$ ) was stirred at $85{ }^{\circ} \mathrm{C}$ for $24 \mathrm{~h}$. The reaction mixture was quenched with $1 \mathrm{~N} \mathrm{HCl}(50 \mathrm{~mL})$ and extracted with AcOEt $(20 \mathrm{~mL} \times 3)$. The combined organic layer was then washed with brine $(50 \mathrm{~mL} \times 2)$ and dried with $\mathrm{MgSO}_{4}$. After removing the solvent in vacuo, the residue was purified by flash column chromatography on silica gel eluted with hexane/AcOEt 50:1 10:1) to give $9(84.8 \mathrm{mg}, 67 \%)$ as pale-yellow solids (mp. 
205.7-205.8 $\left.{ }^{\circ} \mathrm{C}\right): \quad{ }^{1} \mathrm{H}$ NMR $\left(300 \mathrm{MHz}, \mathrm{CDCl}_{3}\right): \delta 1.53(\mathrm{~s}, 6 \mathrm{H}), 3.06(\mathrm{~s}, 4 \mathrm{H}), 3.83(\mathrm{~s}, 4 \mathrm{H}), 7.09(\mathrm{~d}$, $J=16.5 \mathrm{~Hz}, 2 \mathrm{H}), 7.17(\mathrm{~d}, J=16.5 \mathrm{~Hz}, 2 \mathrm{H}), 7.25-7.42(\mathrm{~m}, 7 \mathrm{H}), 7.51-7.55(\mathrm{~m}, 5 \mathrm{H}) ; \quad{ }^{13} \mathrm{C} \mathrm{NMR}$ $\left(75 \mathrm{MHz}, \mathrm{CDCl}_{3}\right): \delta 24.0,39.3,41.4,69.1,98.0,123.6,125.9,126.4,127.6,128.6,129.4,132.9$, 137.4, 139.9; $\quad \mathrm{MS}(\mathrm{EI}): \mathrm{m} / \mathrm{z}(\%): 422(100)\left[\mathrm{M}^{+}\right] ; \quad$ EA calcd $(\%)$ for $\mathrm{C}_{30} \mathrm{H}_{30} \mathrm{O}_{2}$ (422.56): C 85.27, H 7.16; found: C 85.25, H 7.18.

Procedure of Sonogashira Reaction of 4e. A solution of $\mathrm{Pd}\left(\mathrm{PPh}_{3}\right)_{4}(17.4 \mathrm{mg}, 0.015 \mathrm{mmol}), \mathrm{CuI}$ $(5.7 \mathrm{mg}, 0.030 \mathrm{mmol}), 4 \mathrm{e}(141.1 \mathrm{mg}, 0.30 \mathrm{mmol})$, and ethynylbenzene $(92.9 \mathrm{mg}, 0.91 \mathrm{mmol})$ in degassed ${ }^{i} \mathrm{Pr}_{2} \mathrm{NH}(5 \mathrm{~mL})$ was stirred at room temperature for $2 \mathrm{~h}$. The reaction mixture was quenched with $1 \mathrm{~N} \mathrm{HCl}(40 \mathrm{~mL})$ and extracted with AcOEt $(20 \mathrm{~mL} \times 3)$. The combined organic layer was then washed with distilled water $(50 \mathrm{~mL})$ and dried with $\mathrm{MgSO}_{4}$. After removing the solvent in vacuo, the residue was purified by flash column chromatography on silica gel eluted with hexane/AcOEt 50:1 5:1) to give $10(121.1 \mathrm{mg}, 97 \%)$ as colorless solids (mp. 161.6-162.0 $\left.{ }^{\circ} \mathrm{C}\right)$ : ${ }^{1} \mathrm{H}$ NMR (300 MHz, $\left.\mathrm{CDCl}_{3}\right): \delta 1.51(\mathrm{~s}, 6 \mathrm{H}), 3.10$ (s, $\left.4 \mathrm{H}\right), 3.82(\mathrm{~s}, 4 \mathrm{H}), 7.33$ (s, $\left.2 \mathrm{H}\right)$, 7.34-7.39 (m, $6 \mathrm{H})$, 7.53-7.56 (m, $4 \mathrm{H}) ;{ }^{13} \mathrm{C} \mathrm{NMR}\left(75 \mathrm{MHz}, \mathrm{CDCl}_{3}\right): \delta 23.9,40.3,40.9,68.8,87.5,94.1,97.9$, 119.9, 123.0, 128.2, 128.3, 129.5, 131.4, 143.6; MS (EI): $m / z(\%): 418(100)\left[\mathrm{M}^{+}\right], 343(29)\left[\mathrm{M}^{+}\right.$$\left.\mathrm{H}-\mathrm{OC}\left(\mathrm{CH}_{3}\right)_{2} \mathrm{O}\right] ; \quad$ EA calcd (\%) for $\mathrm{C}_{30} \mathrm{H}_{26} \mathrm{O}_{2}$ (418.53): C 86.09, H 6.26; found: C 85.99, H 6.23.

Procedure of Suzuki-Miyaura Coupling of 4e. A solution of $\operatorname{Pd}_{2}(\mathrm{dba})_{3} \cdot \mathrm{CHCl}_{3}(7.8 \mathrm{mg}, 0.0075$ mmol), SPhos (13.7 mg, $0.033 \mathrm{mmol}), 4 \mathrm{e}(141.1 \mathrm{mg}, 0.30 \mathrm{mmol})$, phenylboronic acid (146.6 mg, $1.20 \mathrm{mmol})$, and $\mathrm{K}_{3} \mathrm{PO}_{4}(254.8 \mathrm{mg}, 1.20 \mathrm{mmol})$ in degassed toluene $(1 \mathrm{~mL})$ was stirred at $110{ }^{\circ} \mathrm{C}$ for $24 \mathrm{~h}$. The reaction mixture was quenched with sat. $\mathrm{NH}_{4} \mathrm{Cl}(40 \mathrm{~mL})$ and extracted with $\mathrm{AcOEt}(20$ $\mathrm{mL} \times 3)$. The combined organic layer was then washed with brine $(50 \mathrm{~mL})$ and dried with $\mathrm{MgSO}_{4}$. After removing the solvent in vacuo, the residue was purified by flash column chromatography on silica gel eluted with hexane/AcOEt 20:1 5:1) to give $11(95.5 \mathrm{mg}, 87 \%)$ as pale-yellow solids (mp. 186.5-186.7 $\left.{ }^{\circ} \mathrm{C}\right): \quad{ }^{1} \mathrm{H}$ NMR $\left(300 \mathrm{MHz} \mathrm{CDCl}_{3}\right): \delta 1.42(\mathrm{~s}, 6 \mathrm{H}), 3.06(\mathrm{~s}, 4 \mathrm{H}), 3.75(\mathrm{~s}, 4 \mathrm{H})$, $7.34(\mathrm{~s}, 2 \mathrm{H}), 7.35-7.40(\mathrm{~m}, 2 \mathrm{H}), 7.43-7.52(\mathrm{~m}, 8 \mathrm{H}) ;{ }^{13} \mathrm{C} \mathrm{NMR}\left(75 \mathrm{MHz}, \mathrm{CDCl}_{3}\right): \delta 23.8,40.4$, 
41.9, 68.8, 97.9, 126.9, 127.7, 128.2, 128.5, 137.7, 139.5, 140.6; $\mathrm{MS}(\mathrm{EI}): \mathrm{m} / z(\%): 370(73)\left[\mathrm{M}^{+}\right]$, $355(38)\left[\mathrm{M}^{+}-\mathrm{CH}_{3}\right], 295(100)\left[\mathrm{M}^{+}-\mathrm{H}-\mathrm{OC}\left(\mathrm{CH}_{3}\right)_{2} \mathrm{O}\right]$; $\quad$ EA calcd (\%) for $\mathrm{C}_{26} \mathrm{H}_{26} \mathrm{O}_{2}$ (370.48): C 84.29, H 7.07; found: C 84.37, H 7.02.

Procedure for Synthesis of Penta-p-phenylene 13. A solution of $\mathrm{Pd}_{2}(\mathrm{dba})_{3} \cdot \mathrm{CHCl}_{3}(15.6 \mathrm{mg}$, $0.015 \mathrm{mmol})$, SPhos (27.2 mg, $0.066 \mathrm{mmol}), 4 \mathrm{e}$ (141.4 mg, $0.30 \mathrm{mmol}), 12$ (278.0 mg, $0.90 \mathrm{mmol})$, and $\mathrm{K}_{3} \mathrm{PO}_{4}\left(255.5 \mathrm{mg}, 1.2 \mathrm{mmol}\right.$ ) in degassed mixed solvent (toluene $1 \mathrm{~mL} / \mathrm{H}_{2} \mathrm{O} 10 \mu \mathrm{L}$ ) was stirred at $110{ }^{\circ} \mathrm{C}$ for $15 \mathrm{~h}$. The reaction mixture was quenched with sat. $\mathrm{NH}_{4} \mathrm{Cl}(50 \mathrm{~mL})$ and extracted with AcOEt $(20 \mathrm{~mL} \times 3)$. The combined organic layer was then washed with brine $(50 \mathrm{~mL})$ and dried with $\mathrm{MgSO}_{4}$. After removing the solvent in vacuo, the residue was purified by flash column chromatography on silica gel eluted with hexane/AcOEt 50:1 5:1) to give $13(90.8 \mathrm{mg}, 50 \%)$ as colorless solids (mp. 224.5-225.0 $\left.{ }^{\circ} \mathrm{C}\right): \quad{ }^{1} \mathrm{H} \mathrm{NMR}\left(300 \mathrm{MHz}, \mathrm{CDCl}_{3}\right): \delta 1.42(\mathrm{~s}, 6 \mathrm{H}), 2.91(\mathrm{~s}, 4 \mathrm{H})$, 3.75 (s, 4 H), $5.11(\mathrm{~s}, 4 \mathrm{H}), 5.29$ (s, $4 \mathrm{H}), 7.17$ (s, $2 \mathrm{H}), 7.33$ (d, J= 7.5 Hz, $2 \mathrm{H}), 7.42(\mathrm{~d}, J=7.5 \mathrm{~Hz}$, $2 \mathrm{H}), 7.35-7.51(\mathrm{~m}, 10 \mathrm{H}) ;{ }^{13} \mathrm{C} \mathrm{NMR}\left(75 \mathrm{MHz}, \mathrm{CDCl}_{3}\right): \delta 23.8,40.0,41.8,68.7,73.6,73.8,98.0$, $126.9,127.4,127.6,127.7,128.5,128.6,133.5,134.9,136.1,137.5,138.3,139.8,140.0 ; \quad$ MS (EI): $m / z(\%): 606(91)\left[\mathrm{M}^{+}\right], 591(47)\left[\mathrm{M}^{+}-\mathrm{CH}_{3}\right], 548(100)\left[\mathrm{M}^{+}-\mathrm{OC}\left(\mathrm{CH}_{3}\right)_{2}\right] ; \quad \mathrm{EA}$ calcd (\%) for $\mathrm{C}_{42} \mathrm{H}_{38} \mathrm{O}_{4}$ (606.75): C 83.14, H 6.31; found: C 82.90, H 6.55.

Procedure for Synthesis of Hexa-p-phenylene 17. A solution of $\mathrm{Pd}_{2}(\mathrm{dba})_{3} \cdot \mathrm{CHCl}_{3}(15.4 \mathrm{mg}$, $0.015 \mathrm{mmol}$ ), SPhos (27.2 mg, $0.066 \mathrm{mmol}), 16$ (215.5 mg, $0.30 \mathrm{mmol}), 12$ (277.6 mg, $0.90 \mathrm{mmol})$, and $\mathrm{K}_{3} \mathrm{PO}_{4}\left(254.7 \mathrm{mg}, 1.2 \mathrm{mmol}\right.$ ) in degassed mixed solvent (toluene $1 \mathrm{~mL} / \mathrm{H}_{2} \mathrm{O} 10 \mu \mathrm{L}$ ) was stirred at $110{ }^{\circ} \mathrm{C}$ for $24 \mathrm{~h}$. The reaction mixture was quenched with sat. $\mathrm{NH}_{4} \mathrm{Cl}(40 \mathrm{~mL})$ and extracted with AcOEt $(20 \mathrm{~mL} \times 3)$. The combined organic layer was then washed with brine $(50 \mathrm{~mL})$ and dried with $\mathrm{MgSO}_{4}$. After removing the solvent in vacuo, the residue was purified by flash column chromatography on silica gel eluted with hexane/AcOEt 50:1 1:1) to give 17 (94.4 mg, 37\%) as pale-yellow solid (sublimation around $\left.167{ }^{\circ} \mathrm{C}\right):{ }^{1} \mathrm{H}$ NMR $\left(300 \mathrm{MHz}, \mathrm{CDCl}_{3}\right): \delta 3.55(\mathrm{~s}, 4 \mathrm{H}), 3.59$ (s, $4 \mathrm{H}), 3.74$ (s, $12 \mathrm{H}), 5.14$ (s, $4 \mathrm{H}), 7.18(\mathrm{~d}, J=8.1 \mathrm{~Hz}, 2 \mathrm{H}), 7.22$ (d, J = 8.1 Hz, $2 \mathrm{H})$, 7.34-7.49 $(\mathrm{m}, 14 \mathrm{H}) ;{ }^{13} \mathrm{C}$ NMR $\left(75 \mathrm{MHz}, \mathrm{CDCl}_{3}\right): \delta 40.1,40.3,53.0,60.1,109.1,127.2,127.4,127.7,127.8$, 
$127.9,128.5,128.6,133.3,135.0,135.4,136.1,137.5,138.4,138.7,139.8,171.7 ; \quad$ MS (EI): $m / z$ (\%): 857 (91) $\left[\mathrm{MH}_{2}^{+}\right] ; \quad$ EA calcd (\%) for $\mathrm{C}_{54} \mathrm{H}_{46} \mathrm{O}_{10}$ (854.94): C 75.86, H 5.42; found: C 75.85, H 5.43 . 